\title{
Longitudinal Study
}

National Cancer Institute

\section{Source}

National Cancer Institute. Longitudinal Study. NCI Thesaurus. Code C15273.

A study in which participants with no known disease are initially assessed, and then reassessed for the same characteristic at mutiple timepoints in the future. 